\title{
MAGNETIC FLUX DENSITY AND RADIAL DISTRIBUTION OF CURRENTS IN HTSC SAMPLES OF SPECIFIC SHAPE DETERMINED BY GENETIC ALGORITHM
}

\author{
K. Piotrowski, M.U. Gutowska and M. Gutowski \\ Institute of Physics, Polish Academy of Sciences \\ Al. Lotników 32/46, 02-668 Warsaw, Poland
}

Flux distribution in a $\mathrm{Bi}_{2} \mathrm{Sr}_{2} \mathrm{CaCl}_{2} \mathrm{O}_{8}$ superconductor sample, shaped as a thin disk with a hole in the center, and covered with a thin layer of magnetic garnct, was observed by means of magneto-optical Faraday effect. Applying external magnetic field perpendicularly to the sample plane with zero field cooling history, we were able to observe the flux trapped in superconductor, visible as concentric rings. Both directions of the trapped field were observed near the sample surface. Using the genetic algorithm we were able to determine the radial distribution of concentric currents in the sample under study.

PACS numbers: 74.60.Ge, 74.60.Ec, 74.60.Jg

\section{Introduction}

Flux penetration in plates or thin film of superconductors was presented in several papers [1-3], because it is an interesting and fundamental problem. Flux trapping and flux motion are very important for superconductor applications, especially for high temperature superconductors (IITSC). We believe that commercial applications of high temperature superconductors will make use of HTSC thin films or bulk materials in presence of external magnetic field, especially the field perpendicular to their surface. Such a field may induce electric currents in HTSC samples. A study and analysis of flux penetration and magnetic flux density distribution near the HTSC sample make possible the determination of distribution of corresponding currents inside the sample.

A Faraday technique, with a garnet film as a sensor, was used to determine the $z$-component of $\boldsymbol{B}(x, y)$ - the local magnetic flux density in the $x y$ plane of a sample. The images of magnetic domains were acquired by photo camera and by CCD camera, and digitized by a frame grabber in a PC-type computer.

In our paper we present an experimental study of the magnetic flux penetration into a ring-shaped, high-temperature superconductor, $\mathrm{Bi}_{2} \mathrm{Sr}_{2} \mathrm{CaCu}_{2} \mathrm{O}_{8}$ single crystal. The aim of this paper is the determination of current distribution in HTSC ring from the flux distribution after changes of external magnetic field perpendicular to the ring. The results, obtained by computer simulations using the genetic algorithm, are in very good agreement with experimental data. 


\section{Experiment}

Our measurements were performed using magneto-optical techniques to visualize the flux penetration into a HTSC sample after its special preparation. The complete sample was in form of a sandwich, owning axial symmetry and consisting of a glassy substrate plate, a HTSC ring (a disk with a cylindrical hole in the center) and the cover layer made of disk-shaped garnet film. The dimensions of the HTSC ring were: the inner diameter $2 r_{1}=0.236 \mathrm{~mm}$, the outer diameter $2 r_{2}=0.622 \mathrm{~mm}$ and the thickness $h=0.350 \mathrm{~mm}$. The diameter of the garnet layer was equal to $10 \mathrm{~mm}$ and its thickness was $0.0115 \mathrm{~mm}$. We used the garnet film with stripe domains arranged in a labyrinth pattern. The magnetization vector within domains was oriented normally to the garnet film surface. The saturation level of the garnet film sensor was close to 110 Oe. The sandwich HTSC/garnet film was prepared to be strain free. Soft changes of magnetic domain pattern subjected to low magnetic fields confirmed that this was indeed the case. All the experiments were performed with the sample placed in a continuous flow cryostat Oxford CF-204. Basic software, i.e. MultiScan program, used in our computer system to store/retrieve and process the images, were delivered by CSS Ltd. The source of external magnetic field, applied perpendicularly to the sample's plane, was a pair of Helmholtz coils. The results reported here were obtained at the liquid helium temperature, $4.2 \mathrm{~K}$. We present several results, obtained at various magnitudes of magnetic field acting on the sample, starting from the zero-field cooled (ZFC) state.

\section{Calculations}

The experimental data, serving as an input for calculations, described the radial distribution of magnetic field close to superconductor. The task for computer simulation was to reconstruct the distribution of electric currents in a superconducting ring. The computed distribution of currents had to simulate the experimental distribution of magnetic field near the superconductor in the regions, where our magnetic sensor was saturated, as well as in those regions, where the magnetic domain structure was well resolved. The only two assumptions, we have made at this point, were that only the component $j_{\phi}$ of a current is non-vanishing and that it is a smooth function of $r$ - the distance from the geometric center of a sample. The method used, the smooth genetic algorithm [4], is a very general optimizing procedure, based on a series of transformations applied to a set of many trial solutions of a given problem, at once, in order to improve them. In our case better solutions are those, which generate a better approximation to the experimentally observed distribution of $z$-component of magnetic field near the sample. To be more specific: we introduce the objective function serving as a measure of discrepancy between observed and simulated distributions of a field. This function should be minimized during the calculation course. It is simply an average absolute value of differences between simulated and observed $z$-components of magnetic field per degree of freedom. We define the degree of freedom as a number of experimental points, for which either the simulated value of a field exceeds the saturation field of a sensor and the sensor is not saturated or the magnitude of simulated field does not exceed the saturation field of a sensor. Other points, i.e. those with saturated sensor and simulated magnitude of magnetic field exceeding the saturation 
field of magnetic cover layer, do not contribute to the objective function (iff, additionally, both fields have the same sign). The demagnetizing field, generated by non-uniformly magnetized sensor (and usually neglected), was taken into account at every step of calculations.
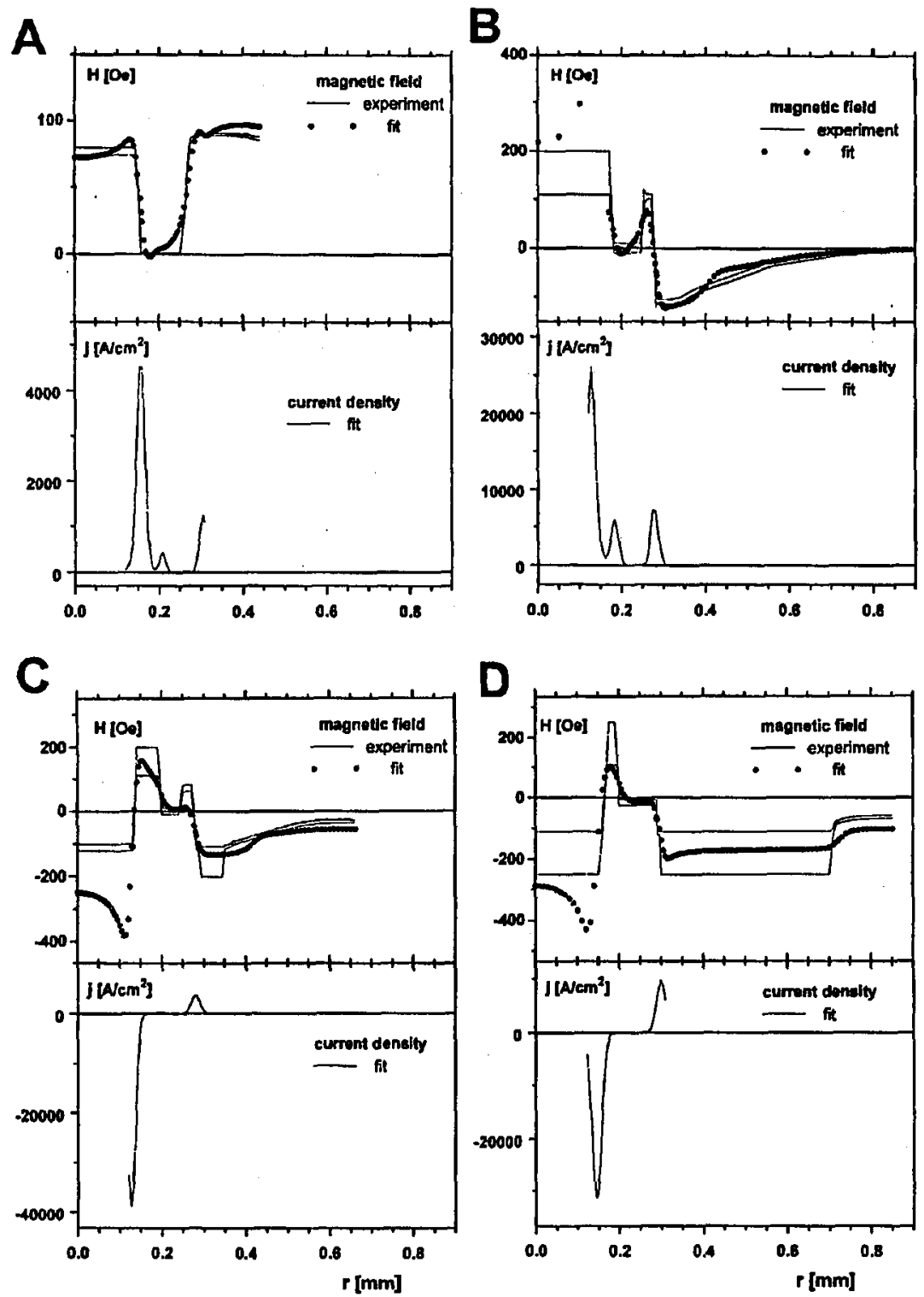

Fig. 1. Measured and simulated distributions of magnetic field near superconducting ring with corresponding radial distributions of currents as a result of computer modeling in an external field $H_{\text {ext }}=(\mathrm{A}) 87 \mathrm{Oe}$, (B) $0 \mathrm{Oe},(\mathrm{C})-30 \mathrm{Oe}$, (D) $-63 \mathrm{Oe}$. 


\section{Results}

The radial distributions of currents obtained as a result of computer modeling, as well as the simulated and measured distributions of magnetic field near the superconducting ring, are shown in Fig. 1.

Figure 1a shows the situation with external field introduced after ZFC cycle. The consecutive figures $b, c$ and $d$ present more results obtained after ZFC cycle. In Fig. $1 \mathrm{~b}$ the magnetic field was reduced to zero after reaching first the value of $H_{\max }=900 \mathrm{Oe}$. Next, the sign of field was reversed and the field was increased again. Figures $1 \mathrm{c}$ and $1 \mathrm{~d}$ show this evolution as a variation in currents' distribution caused by the change of external magnetic field.

It should be stressed that the obtained distributions of current, and their changes induced by changes of external magnetic field, are qualitatively consistent with existing basic theoretical models [5]. The quantitatively agreement with other author's results is also noticeable.

\section{Summary}

The application of an optical system, which joins features of two kinds of microscopes, namely the transmissive and reflective one, makes possible to observe, and evaluate, the distribution of magnetic flux just above the surface of the superconducting ring, within the hole and around its edges.

Taking into account the demagnetizing field of a magnetized garnet layer greatly improved the shapes of simulated magnetic field distributions in regions far from the superconducting ring.

The simulation procedure (the genetic algorithm) does not require earlier knowledge of an analytical, or any other, form of future solutions. Nevertheless, the solutions, it delivers, are consonant with theoretical models.

\section{Acknowledgments}

The authors thank Prof. A. Balbashov for the single-crystalline sample used in the described measurements. This work was supported by the Committee for Scientific Research (grant No. 2P 302082 04).

\section{References}

[1] A. Forkl, H.U. Habermeier, B. Leibold, T. Dragon, H. Kronmüller, Physica C 180, 155 (1991).

[2] L.T. Baczewski, K. Piotrowski, R. Szymczak, H. Szymczak, A.P. Malozemoff, Physica $C$ 175, 363 (1991).

[3] P.D. Grant, M.W. Denhoff, W. Xing, P. Brown, S. Govorkov, J.C. Irwin, B. Heinrich, H. Zhou, A.A. Fife, A.R. Cragg, Physica C 229, 289 (1994).

[4] M.W. Gutowski, J. Phys. A, Math. Gen. 27, 7893 (1994).

[5] A.C. Rose-Innes, E.H. Rhoderick, Introduction to Superconductivity, Pergamon Press, 1969, Chap. 2. 